\title{
THE 'HAMMOCK' EFFECT IN WHEELCHAIR CUSHION COVERS
}

\author{
By W. A. Denne, B.Sc., Ph.D. \\ Charleston House, Cudlow Avenue, Rustington, West Sussex
}

\begin{abstract}
It is necessary to cover wheelchair cushions in order to protect the load distributing elements from wear and contamination. It is widely supposed that the more rigid covers may impair the load distributing properties of the cushion by the so-called 'hammock' effect. Measurements have therefore been taken of cushion deformation and seating pressures using a standard Department of Health wheelchair cushion with a variety of covers to determine the degree of impairment. The results indicate that the cushion cover 'hammock' effect may be ignored for practical purposes.
\end{abstract}

Key words: Wheelchair cushion covers; Pressure sore prophylaxis; Hammock effect.

IT is of great importance to maintain low pressures between wheelchair patients and their seating systems, as high pressures sustained for long periods prevent blood flow which in turn produces soft tissue necrosis and pressure sores. Wheelchair cushioning material is therefore carefully selected to produce low seating pressures. However, it is necessary to cover these cushions in order to minimise wear and soiling of the load distributing element and to facilitate cleansing. It is widely considered that such covers may impair the functioning of wheelchair cushions especially in the crucial regions of high pressure.

The reasons for this are twofold. The PVC laminated fabrics which are commonly used for wheelchair cushion covers feel hard and inextensible to the touch. Comparison with the soft appearance of polyurethane foam leads one intuitively to suppose that the cover will make the cushion harder. A more scientific approach suggests that a substantial 'hammock' effect may be generated. This effect is simply an expression of the restoring force produced when a tensioned membrane is indented. The support obtained when sitting in a hammock is an obvious but somewhat misleading example of this phenomenon.

It is well known that a tensioned curved membrane produces a radial pressure which is proportional to the tension and degree of curvature. In a wheelchair cushion cover, the maximum curvature generally lies under the ischial tuberosities and if the cover is tensioned, the greatest additional pressure will be produced in this sensitive region. However, the cushion cover is not tethered at its extremities like a hammock. Tension can only be generated by friction between the cover and the cushion and by the tangential in-plane compressive forces within the cover which prevent it flowing to accommodate a curved surface. Cushion cover tension may therefore arise, though in a somewhat different fashion to a hammock, and this may produce increased seating pressures. The crucial question is how large will the effect be under clinical conditions.

Preliminary measurements (Ring \& Urquhart, 1979) have suggested that the effect may not be as great as has been supposed. It was therefore considered worthwhile to conduct more systematic tests using a range of covers of varying degrees of rigidity to establish whether cushion cover 'hammock' effects are in fact a serious problem in wheelchair seating. 


\section{Experimental Studies}

The tests consisted of seating subjects in a standard posture on a standard cushion with a succession of different covers. When equilibrium under load was attained, the pressure under the left ischial tuberosity was measured together with an index of indentation of the cushion and an estimate of the degree of stretching of the cushion cover.

The cushion used was a standard Department of Health and Social Security (DHSS) polyurethane foam insert measuring $40 \times 40 \times 7.5 \mathrm{cms}$. It was used without the customary muslin cover to provide the worst case for cushion/cover friction. The cushion was placed in a seating jig to provide reproducibility of sitting posture. The jig had a flat plywood base, lateral hip locating blocks, a vertical backboard to locate the posterior aspect of the pelvis and a goad fitted centrally in the backboard at mid-thoracic level. Sensation of the goad on the centreline of the back ensured that the subject was vertical. The subject was seated in this jig with muscles relaxed, no supportive loading on the feet, and with hands placed loosely in the lap. Initial trials showed that despite the sensitivity of ischial tuberosity pressures to posture, the reproducibility of sitting posture was adequate for the present purpose.

The positions of the ischial tuberosities with respect to a laboratory frame of axes were determined by palpation and the coordinates were recorded on the seating jig. A length of cotton was stretched taut across the line joining the ischial tuberosity positions and was retained on both sides of the cushion and on both sides of the jig by self adhesive tags (Fig. I). The cotton was marked at each of these four points prior to seating the subject. A pneumatic pressure sensor developed at this Centre was placed at the coordinates of the left ischial tuberosity and the subjects were sat repeatedly on the cushion with a succession of six different covering arrangements.

For each cover three independent assessments of pressure were made to provide an estimate of reproducibility. Two independent measures were taken of the degree of cotton pull-through due to seat cover extension and indentation of the cover.

Two healthy volunteer subjects were used, a $79 \mathrm{~kg}$ male and a $62 \mathrm{~kg}$ female, as the test regime was considered too taxing for a disabled person.

The cushion covering arrangements tested were no cover at all, then covers made from Platylon (a $0.05 \mathrm{~mm}$ thick polyurethane film), a relatively rigid synthetic fabric used for covering aircraft seating, a two way stretch synthetic fabric with a heat-sealed highly extensible plastic film backing, a soft and extensible foamed PVC sheet moulded onto a fabric backing, and last, the standard DHSS PVC cover. The covers were ranked in order of apparent increase in rigidity and the tests were made in this sequence. They were followed by a repeat of the no-cover pressure measurements to check on drift and reproducibility of the pressure sensor. The experimental data are presented in Table $\mathrm{I}$.

\section{Results and Discussion}

The most striking result is the virtual absence of any change in ischial tuberosity pressure with the increase in cover stiffness. The mean of the r.m.s. deviations from the mean for the sets of three pressure readings is $3.0 \mathrm{~mm} \mathrm{Hg}$. This implies that the readings taken at the beginning and end of each run indicate 


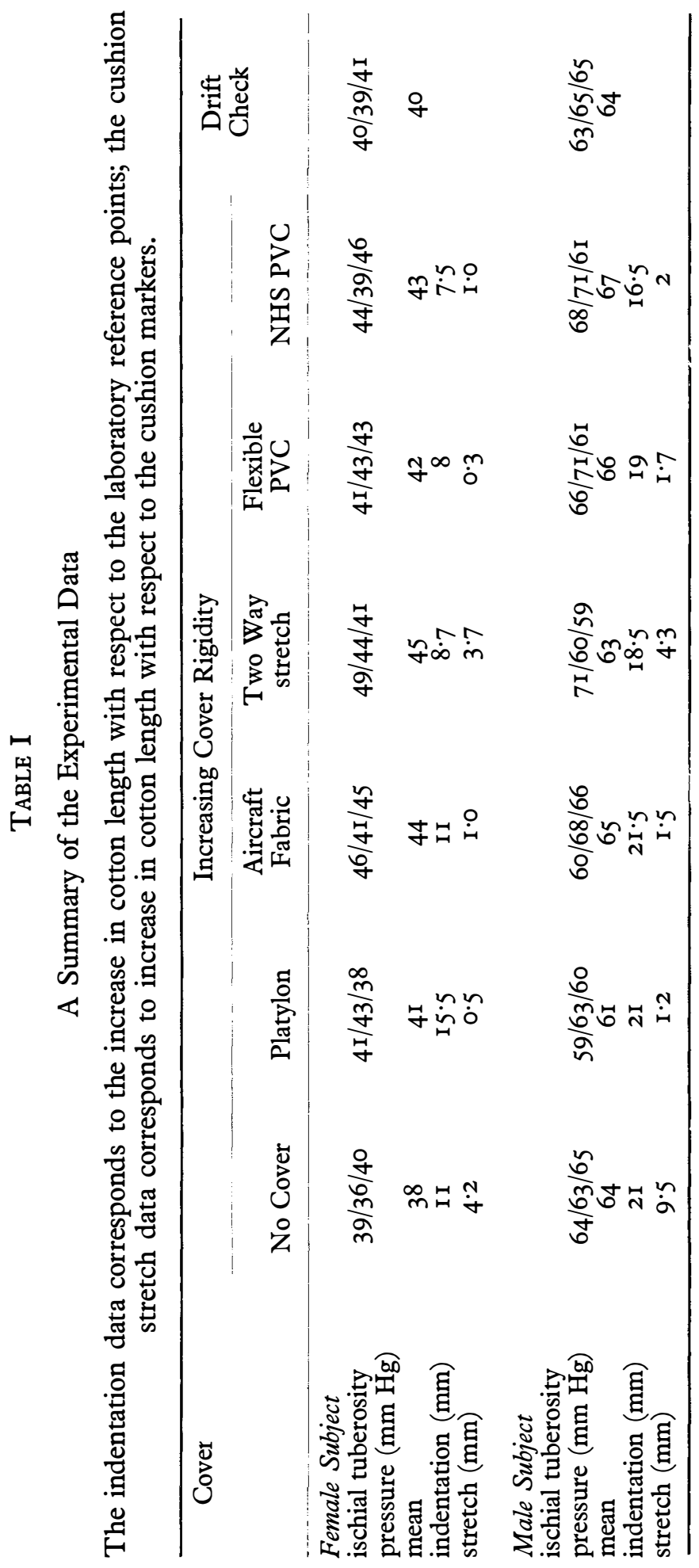




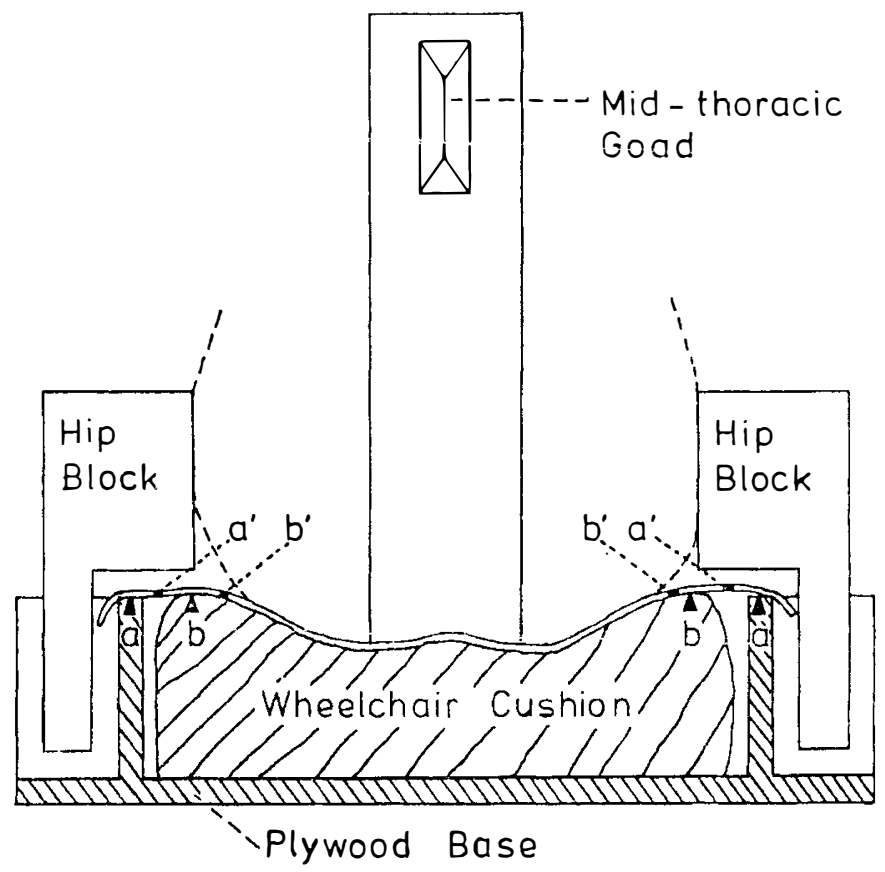

FIG. I

The cushion cover and laboratory reference system. The points, a, represent the laboratory frame of reference and the points, $a^{\prime}$, on the cotton line up with these before seating the subject. The difference $a-a^{\prime}$ after sitting provides an index of indentation of the cushion. Similarly, the points, $b$, provide a cushion cover frame of reference, the marks, $b^{\prime}$, being aligned with them prior to sitting. After a test, the difference $b-b^{\prime}$ represents the degree of stretch in the cover during sitting.

no significant drift in the pressure sensor. There is no significant difference between the pressure readings taken under the male subject but the readings from the female subject indicate a 'hammock' effect which is just significant. The largest increase in pressure is for the two way stretch fabric, being 13 per cent above the mean baseline pressure. Since much larger changes in ischial tuberosity pressure can be caused by slight changes in posture or small alterations to the seating arrangement, it is reasonable to conclude that the 'hammock' effect in wheelchair cushion covers is not of clinical significance for covers which are no more rigid than the current DHSS model.

A closer look at the results yields further information of interest. Apart from the two way stretch fabric, no appreciable cover extension is observed under load for either subject, though as one might expect, the extensions are systematically greater for the heavier subject. Contrary to expectation, the deformation of the two way stretch fabric did not result in lower pressures at the ischial tuberosity.

It is intriguing that the indentation reduces with stiffening cushion covers, so there is a significant change in the degree of conformation of a cushion when it is covered with a DHSS style of cover. The sensation of this difference is often observed by patients. As observed previously, however, this is of little clinical significance from a pressure reduction viewpoint. 


\section{SUMMARY}

An experimental investigation has shown that the 'hammock' effect in wheelchair cushion covers is insufficient under most clinical conditions to adversely affect the seating pressure distribution.

\section{RÉSUMÉ}

Cette étude indique que la pression posée par les coussins de voiture de malade est pour la plupart indépendante de la forme de couverture employée pour les coussins.

\section{ZUSAMMENFASSUNG}

Diese Studie zeigt, dass die Art der Kissenbezüge auf den Sitzdruck der Rollstuhlkissen kaum Einfluss hat.

Acknowledgements. The author would like to thank Dr J. Reid, Technical Manager of Declon Plastics, Dunstable, for the loan of the non-standard cushion covers which he has developed for use with the orthaton wheelchair cushion. Thanks are also due to Miss Rosemary Merivale who kindly volunteered as a subject for the experimental work, and to members of staff of the Mary Marlborough Lodge for their helpful collaboration.

\section{REFERENCE}

RING, N. D. \& URQuhaRT, D. Private communication. 